\title{
Wave energy and spatial variability in community structure of small cryptic coral reef fishes
}

\author{
Martial Depczynski*, David R. Bellwood \\ Centre for Coral Reef Biodiversity, Department of Marine Biology, James Cook University, Townsville 4811, Australia
}

\begin{abstract}
The distribution and abundance of small cryptic reef fishes were quantified among microhabitats and reef zones at both exposed and sheltered sites at Lizard Island, Great Barrier Reef. A total of 1042 individuals from 44 species in 8 families were sampled. Marked variation in abundance, species numbers, size-class distribution and species composition were displayed among reef zones at exposed sites; in contrast, comparatively little variation was found in sheltered sites. The exposed, wave-swept front flat reef zone was clearly the most depauperate and species-poor zone with calmer back reef, lagoonal and reef base zones containing the highest numbers of individuals and species. Larger, heavier individuals predominated in exposed wave-swept reef zones whereas smaller, lighter individuals were commonly found in calmer waters. Sheltered sites exhibited little variation among zones. In these sites, microhabitat appeared to play a much greater role in shaping community composition. Overall, $68.5 \%$ of all individuals (70.1\% at exposed and $65.4 \%$ at sheltered sites) were collected from sand/rubble microhabitats as opposed to $31.5 \%$ from open reef microhabitats. Assemblages at exposed and sheltered sites exhibited little taxonomic overlap, even of highly abundant species. These results indicate that wave energy plays a significant role in structuring small cryptic coral reef fish communities, with microhabitat type playing a key role in the absence of wave energy.
\end{abstract}

KEY WORDS: Cryptic - Distribution · Reef fish · Habitat · Community ecology · Gobiidae · Blenniidae · Wave exposure

\section{INTRODUCTION}

Of all reef fishes, the community ecology of small cryptic assemblages are least well understood. Difficulties associated with accurately censusing these hidden fishes (see Brock 1982, Willis 2001, Edgar et al. 2004) have limited our overall understanding of the dynamics and role of reef fish communities on coral reefs. Recent studies using anaesthetics and ichthyocides have shown that small cryptic fishes $(<10 \mathrm{~cm})$ comprise up to half of the fish numbers on coral reefs (Ackerman \& Bellwood 2000, 2002, Greenfield 2003), and constitute a diverse community containing many highly specialised species (e.g. Munday et al. 2002, Depczynski \& Bellwood 2004, 2005, Hobbs \& Munday 2004). Although occupying the lower end of the size spectrum in reef fishes, this group may provide impor- tant insights into the role of fishes in ecosystems, as it is often the smallest organisms that are most abundant, diverse and influential in ecosystem processes (May 1978, Begon et al. 1996). However, we currently lack a detailed description of their distribution and abundance at a reef-wide whole ecosystem scale, an essential pre-requisite in unravelling their contribution to reef ecosystem processes.

Published descriptions of small reef fishes have emphasised that species within this group display marked spatial variation at very small spatial scales of centimetres to metres (Luckhurst \& Luckhurst 1978, Townsend \& Tibbetts 2000, Wilson 2001). Many also exhibit restricted, and often obligate relationships with particular microhabitat types (Munday 2000, Webster \& Hixon 2000, Goncalves et al. 2002, Depczynski \& Bellwood 2004). At an individual level, limited home 
and foraging ranges of less than $2 \mathrm{~m}^{2}$ are consistently reported (Luckhurst \& Luckhurst 1978, Reavis 1997, Goncalves \& Almada 1998, Depczynski \& Bellwood 2004). Despite these studies, nothing is known of the among reef zone variation in small cryptic communities (but see Greenfield \& Johnson 1990, 1999 for family-level studies), the level at which most reef fish communities exhibit the greatest variation in composition and abundance (Williams 1982, 1991, Russ 1984). These small-scale observations suggest that the distribution patterns of small cryptic reef fish communities are also likely to display significant variation at larger, among-zone, spatial scales. At these larger amongzone scales, coral reef assemblages are shaped by physical forces acting either directly on individuals, or through indirect influences on habitat or food availability (Fulton et al. 2001, Gust et al. 2001). Given the small size and benthic associations of the small cryptic coral reef fish community, one may hypothesise that these species are highly likely to exhibit marked zonation along these energy gradients. In this study therefore, we describe and quantify the abundance, size composition and community structure of small cryptic coral reef fishes across a gradient of wave exposure to provide a basis for evaluating the role of these fishes in reef processes.

\section{MATERIALS AND METHODS}

Sampling was undertaken in January and February 2003 on reefs around the Lizard Island group $\left(14^{\circ} 40^{\prime} \mathrm{S}, 145^{\circ} 27^{\prime} \mathrm{E}\right)$ in the Cairns section of the Great Barrier Reef (GBR). Lizard Island is a granite island in the mid-shelf region of the GBR. Three sites were censused on the exposed reef front between Bird and South Isles at depths of 1 to $15 \mathrm{~m}$, and 3 from the sheltered NW margin of Watsons Bay at depths of 1 to $6 \mathrm{~m}$ (Fig. 1). Five reef zones were censused at each site (base, slope, crest, front flat \& back flat) at the exposed reef (Fig. 2). The lack of a defined slope at the sheltered reef sites meant that only 4 reef zones could be reliably identified (base, crest, front flat and back flat). Descriptions of reef zones and corresponding wave energy are given (Table 1). In each reef zone, 4 samples were taken in each of 2 microhabitats, open reef and sand/rubble with a total of 120 censuses at the exposed sites and 96 at the sheltered sites. Open reef microhabitats were flat, open areas of live and/or dead coral fully exposed to the surround-

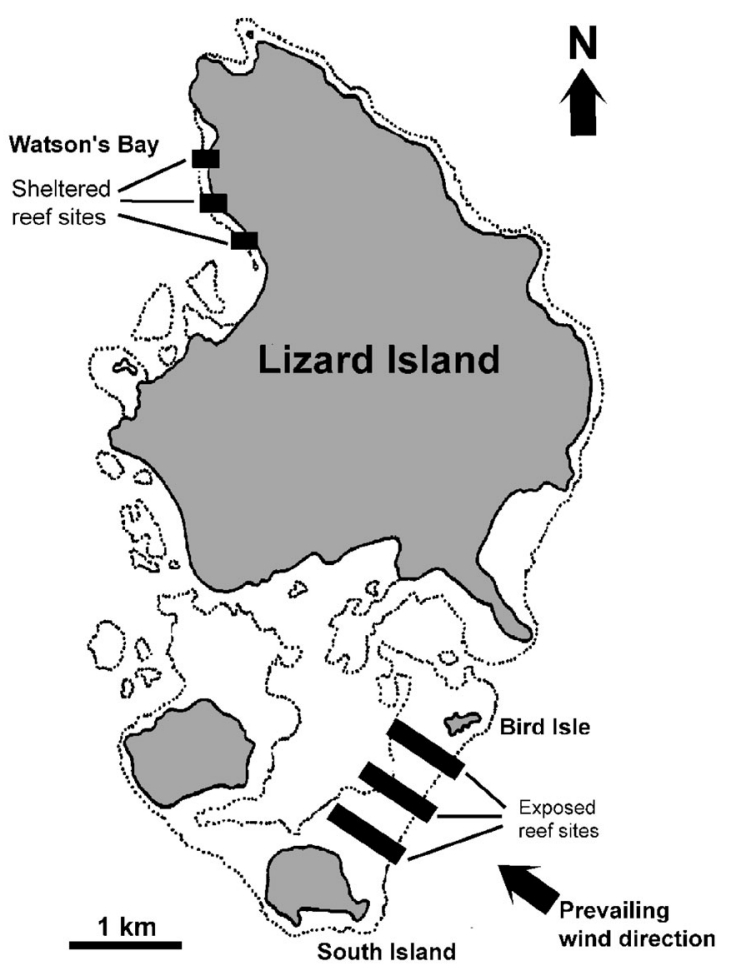

Fig. 1. Exposed and sheltered reef sites where the study was conducted at Lizard Island showing spatial scale and prevailing south-easterly wind direction (modified after Fulton \& Bellwood 2005)

ing water column from all sides and above; sand/rubble areas of sand and hard coral rubble where $>50 \%$ of sample area contained visible coral rubble pieces of between 20 to $200 \mathrm{~mm}$.

Samples were collected on SCUBA using clove oil and a fine-mesh $(2 \mathrm{~mm})$ net covering a basal area of $0.4 \mathrm{~m}^{2}$. The weighted net was positioned in a circle on the substratum before approximately $125 \mathrm{ml}$ of a 5:1 ethanol:clove oil mixture was sprayed into the netted area and left for 1 min before the search for anaes-

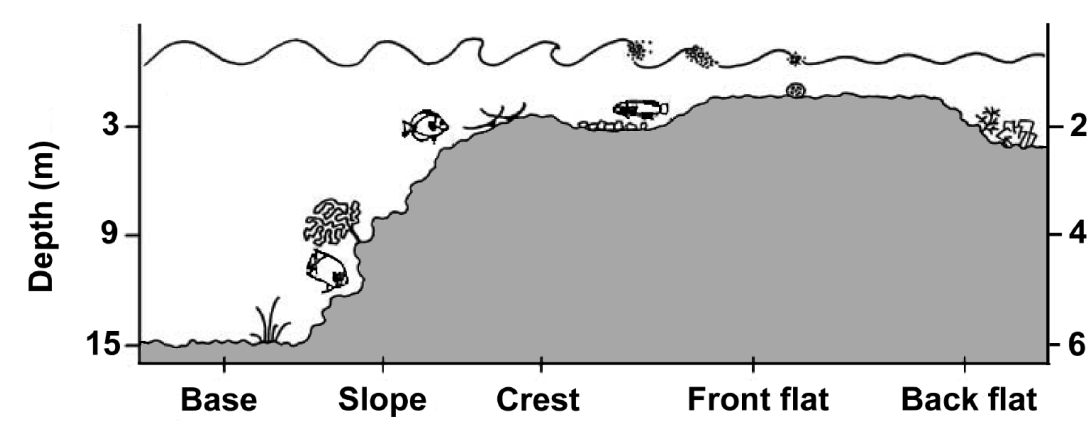

Fig. 2. Reef zones and depth along the reef profile (modified after Fulton \& Bellwood 2005). Depths on the left-hand $y$-axis are for exposed reef sites; right-hand $y$-axis for sheltered reef sites 
Table 1. Environment description, depth and wave severity of reef zones at exposed and sheltered reef sites. Wave energy estimates follows Fulton \& Bellwood (2005) at same location

\begin{tabular}{|c|c|c|c|c|c|}
\hline \multirow[t]{2}{*}{ Reef zone } & \multicolumn{3}{|c|}{ Environment description } & \multicolumn{2}{|c|}{ Physical parameters } \\
\hline & General characteristics & Heterogeneity & Coral cover & Depth $(\mathrm{m})$ & Wave energy \\
\hline \multicolumn{6}{|c|}{ Exposed reef } \\
\hline Base & $\begin{array}{l}\text { Gently sloping fine sand and rubble } \\
\text { environment with isolated coral colonies } \\
\text { or outcrops }\end{array}$ & Moderate & Moderate & $10-15$ & Nil \\
\hline Slope & $\begin{array}{l}\text { Variable incline }\left(10 \text { to } 90^{\circ}\right) / \\
\text { diverse topography }\end{array}$ & Highest & High & $6-9$ & Moderate \\
\hline Crest & High vertical relief & High & Highest & $1-3$ & High \\
\hline Front flat & $\begin{array}{l}\text { Mostly hard flat substrata covered in } \\
\text { algal and coarse sand with occasional } \\
\text { coral outcrops }\end{array}$ & Lowest & Low & $2-4$ & Highest \\
\hline Back flat & Flat, varied environment & Moderate & Moderate & $2-4$ & Moderate \\
\hline \multicolumn{6}{|c|}{ Sheltered reef } \\
\hline Base & $\begin{array}{l}\text { Flat sand and rubble environment } \\
\text { with isolated reef outcrops }\end{array}$ & Lowest & Low & $4-6$ & Nil \\
\hline Crest & High vertical relief & Highest & High & $1-3$ & Low \\
\hline Front flat & Mostly flat, hard substrata environment & Moderate & Moderate & $2-4$ & Low \\
\hline Back flat & Flat, varied environment & Moderate & Moderate & $1-3$ & Low \\
\hline
\end{tabular}

thetised fish began. Following a 5 min systematic search by 2 divers, fish were put into labelled, clip-seal plastic bags, and placed into an ice-water slurry. Specimens were identified, weighed and measured (total length; TL) at the laboratory, and stored in $70 \%$ ethanol. The genus Eviota are small and taxonomically challenging to identify (Lachner \& Karnela 1980). Thus, identification of highly abundant Eviota species were made by H. K. Larson of the Museum and Art Gallery of the Northern Territory. All other Eviota species were provisionally identified to Sp. A, Sp. B. etc based on a combination of meristics, their cephalic sensory pore system and readily distinguishable body and facial markings; the key recognised traits for the identification of this genus (see Lachner \& Karnella 1980). Already numbering some 70 odd species, the samples probably included several undescribed Eviota species.

After initial examination, data were $\log _{10}(x+1)$ transformed to satisfy requirements for normality and homoscedascity. Following $t$-tests comparing overall numbers of individuals and species at exposed and sheltered reef locations, differences in the abundances of individuals and number of species among sites, zones and microhabitats were analysed using 3-way mixed factorial ANOVAs at exposed and sheltered reef locations separately. Variation in species assemblages among zones were examined using MANOVAs based on the 12 most abundant species (with $>10$ individuals across all zones) for exposed and sheltered reef locations separately. Bonferroni-corrected multiple comparisons tests were used to identify differences in species assemblages between zones. Canonical discriminant analyses (CDA) based on structure coefficients graphically identified the characteristics of species assemblages in the 4 (sheltered) and 5 (exposed) reef zones. Zone centroids are displayed with 95\% confidence clouds. Abundance within each species is displayed by the relative size of species points on the CDA following square root transformation on raw counts. Differences in size (using weight in g) of individuals were investigated among zones at exposed and sheltered reef locations separately using 1-way ANOVAs followed by Tukey's HSD post-hoc tests to identify where differences lay.

\section{RESULTS}

\section{Patterns of individual and species abundance}

A total of 1042 individuals from 44 species in 8 families were sampled over a combined area of $86.4 \mathrm{~m}^{2}$ $\left(216 \times 0.4 \mathrm{~m}^{2}\right)$ (Table 2). Of these, the top 19 species (>10 individuals sampled) represented $92.5 \%$ of all individuals, with the Gobiidae making up $87.1 \%$ of all individuals, and tripterygiids, blenniids and pseudochromids contributing most of the remaining $12.9 \%$. While the Gobiidae dominated all reef zones at sheltered and exposed reef sites, differences in the proportional contribution of non-gobiid families among reef zones varied considerably, particularly at exposed reef sites (Fig. 3). 
Table 2. Families (8 total), species, numbers of individuals and numerical and biomass contribution (\%) of each species to the entire assemblage sampled at exposed $(n=120)$ and sheltered $(n=96)$ reef sites. The 19 most abundant species (in bold) represent $92.5 \%$ of all individuals sampled and were chosen for further analyses based on their presence ( $>10$ individuals across all reef zones) at either exposed and/or sheltered reef sites

\begin{tabular}{|c|c|c|c|c|c|c|c|c|c|}
\hline \multirow[t]{2}{*}{ Family } & \multirow{2}{*}{ Species } & \multicolumn{4}{|c|}{ Abundance } & \multicolumn{4}{|c|}{ Biomass (g) } \\
\hline & & $\begin{array}{c}\text { Ex- } \\
\text { posed }\end{array}$ & $\begin{array}{l}\text { Shel- } \\
\text { tered }\end{array}$ & $\begin{array}{c}\text { Total } \\
(\mathrm{T})\end{array}$ & $\%$ of $\mathrm{T}$ & $\begin{array}{c}\text { Ex- } \\
\text { posed }\end{array}$ & $\begin{array}{l}\text { Shel- } \\
\text { tered }\end{array}$ & $\begin{array}{c}\mathrm{T} \\
\text { biomass }\end{array}$ & $\begin{array}{r}\% \text { of } \mathrm{T} \\
\text { biomass }\end{array}$ \\
\hline \multirow[t]{29}{*}{ Gobiidae } & Eviota sigillata & 192 & 24 & 216 & 20.73 & 6.12 & 0.69 & 6.81 & 4.54 \\
\hline & Eviota queenslandica & 99 & 41 & 140 & 13.44 & 3.88 & 1.34 & 5.22 & 3.48 \\
\hline & Eviota melasma & 60 & 21 & 81 & 7.77 & 3.14 & 1.59 & 4.73 & 3.15 \\
\hline & Asterropteryx semipunctatus & 2 & 72 & 74 & 7.1 & 0.15 & 9.08 & 9.23 & 6.15 \\
\hline & Istigobius goldmanni & 31 & 33 & 64 & 6.14 & 5 & 5.04 & 10.04 & 6.69 \\
\hline & Eviota sp. Q & 5 & 34 & 39 & 3.74 & 0.36 & 2.76 & 3.12 & 2.08 \\
\hline & Eviota sp. J & 32 & $\mathbf{0}$ & 32 & 3.07 & 0.78 & $\mathbf{0}$ & 0.78 & 0.52 \\
\hline & Eviota sp. K & 26 & 6 & 32 & 3.07 & 0.87 & 0.18 & 1.05 & 0.7 \\
\hline & Amblyeleotris sp. A & 8 & 17 & 25 & 2.4 & 2.7 & 0.39 & 3.09 & 2.06 \\
\hline & Eviota pellicuda & 25 & $\mathbf{0}$ & 25 & 2.4 & 0.95 & $\mathbf{0}$ & 0.95 & 0.63 \\
\hline & Eviota sp. O & 21 & 3 & 24 & 2.3 & 1.45 & 0.29 & 1.74 & 1.16 \\
\hline & Callogobius sp. A & $\mathbf{0}$ & 20 & 20 & 1.92 & $\mathbf{0}$ & 1.48 & 1.48 & 0.99 \\
\hline & Ctenogobiops feroculus & $\mathbf{0}$ & 19 & 19 & 1.82 & $\mathbf{0}$ & 2.22 & 2.22 & 1.48 \\
\hline & Fusigobius signipinnis & 15 & 2 & 17 & 1.63 & $\mathbf{0}$ & 1.13 & 0.21 & 0.14 \\
\hline & Amblygobius phaelena & 4 & 12 & 16 & 1.54 & 2.6 & 4.04 & 6.64 & 4.42 \\
\hline & Valenciennea muralis & 7 & 9 & 16 & 1.54 & 10.59 & 2.98 & 13.57 & 9.04 \\
\hline & Callogobius sclateri & 3 & 4 & 7 & 0.67 & 1.7 & 1.31 & 3.01 & 2.01 \\
\hline & Eviota sp. S & 1 & 5 & 6 & 0.58 & 0.01 & 0.39 & 0.4 & 0.27 \\
\hline & Istigobius rigilius & 2 & 3 & 5 & 0.48 & 0.84 & 0.46 & 1.3 & 0.87 \\
\hline & Amblygobius rainfordi & 2 & 1 & 3 & 0.29 & 1.53 & 0.39 & 1.92 & 1.28 \\
\hline & Ctenogobiops pomastictus & 0 & 3 & 3 & 0.29 & 0 & 0.56 & 0.56 & 0.37 \\
\hline & Eviota sp. N & 0 & 3 & 3 & 0.29 & 0 & 0.16 & 0.16 & 0.11 \\
\hline & Eviota sp. P & 0 & 3 & 3 & 0.29 & 0 & 0.08 & 0.08 & 0.05 \\
\hline & Signigobius biocellatus & 1 & 2 & 3 & 0.29 & 0.03 & 0.34 & 0.37 & 0.25 \\
\hline & Trimma striata & 3 & 0 & 3 & 0.29 & 0.29 & 0 & 0.29 & 0.19 \\
\hline & Amblygobius nocturnis & 0 & 2 & 2 & 0.19 & 0 & 0.59 & 0.59 & 0.39 \\
\hline & Coryphopterus neophytus & 0 & 2 & 2 & 0.19 & 0 & 1.75 & 2.88 & 1.92 \\
\hline & Gobiodon quinquistregatus & 0 & 2 & 2 & 0.19 & 0 & 1.09 & 1.09 & 0.73 \\
\hline & Eviota sp. F & 1 & 0 & 1 & 0.1 & 0.04 & 0 & 0.04 & 0.03 \\
\hline \multirow[t]{8}{*}{ Blenniidae } & Salarias patzneri & 30 & 24 & 54 & 5.18 & 13.78 & 16.45 & 30.23 & 20.14 \\
\hline & Ecsenius stictus & 5 & 3 & 8 & 0.77 & 3.37 & 2.67 & 6.04 & 4.02 \\
\hline & Salarias guttatus & 2 & 3 & 5 & 0.48 & 3.8 & 2.65 & 6.45 & 4.3 \\
\hline & Entomacrodus sp. A & 0 & 4 & 4 & 0.38 & 0 & 0.92 & 0.92 & 0.61 \\
\hline & Ecsenius bicolour & 2 & 2 & 4 & 0.38 & 1.89 & 4.27 & 6.16 & 4.1 \\
\hline & Crossalarias macrospilus & 1 & 1 & 2 & 0.19 & 0.54 & 1.02 & 0.54 & 0.36 \\
\hline & Salarias fasciatus & 1 & 1 & 2 & 0.19 & 0.14 & 0.41 & 0.55 & 0.37 \\
\hline & Istiblennius sp. A & 0 & 1 & 1 & 0.1 & 0 & 0.31 & 0.31 & 0.21 \\
\hline \multirow[t]{2}{*}{ Apogonidae } & Apogon cooki & 3 & 0 & 3 & 0.29 & 1.82 & 0 & 1.82 & 1.21 \\
\hline & Apogon doederleini & 0 & 1 & 1 & 0.1 & 0 & 0.16 & 0.16 & 0.11 \\
\hline Pseudochromidae & Pseudochromis fuscus & 10 & 2 & 12 & 1.15 & 3.74 & 0.91 & 4.65 & 3.1 \\
\hline Tripterygiidae & Enneapterygius tutuilae & 27 & 31 & 58 & 5.57 & 3.27 & 1.84 & 5.11 & 3.4 \\
\hline Syngnathidae & Corythoichthys flavofasciatus & 2 & 0 & 2 & 0.19 & 0.16 & 0 & 0.16 & 0.11 \\
\hline Pinguipedidae & Parapercis xanthozona & 2 & 0 & 2 & 0.19 & 3.2 & 0 & 3.2 & 2.13 \\
\hline Muraenidae & Sp. A & 1 & 0 & 1 & 0.1 & 0.24 & 0 & 0.24 & 0.16 \\
\hline Total & & 626 & 416 & 1042 & 100 & 78.98 & 71.94 & 150.11 & 100 \\
\hline
\end{tabular}

\section{Exposed versus sheltered reefs}

Overall, exposed and sheltered reefs were broadly similar and statistically non-significant in both mean individual abundance (exposed 14.6 ind. $\mathrm{m}^{-2}$ [ $\pm 1.4 \mathrm{SE}$ ], sheltered 12.5 ind. $\mathrm{m}^{-2}[ \pm 0.9 \mathrm{SE}][t=0.84, \mathrm{df}=214, \mathrm{p}>$
0.05]) (Fig. 4) and mean species numbers (exposed 6.7 ind. $\mathrm{m}^{-2}[ \pm 0.4 \mathrm{SE}]$, sheltered 7.8 ind. $\mathrm{m}^{-2}[ \pm 0.5 \mathrm{SE}]$ [ $t=-1.83, \mathrm{df}=214, \mathrm{p}>0.05]$ ) (Fig. 5). Species richness tended to mirror individual abundances among reef zones and habitats. A total of 31 species were recorded on exposed and 36 species on sheltered reefs (Table 3 ). 


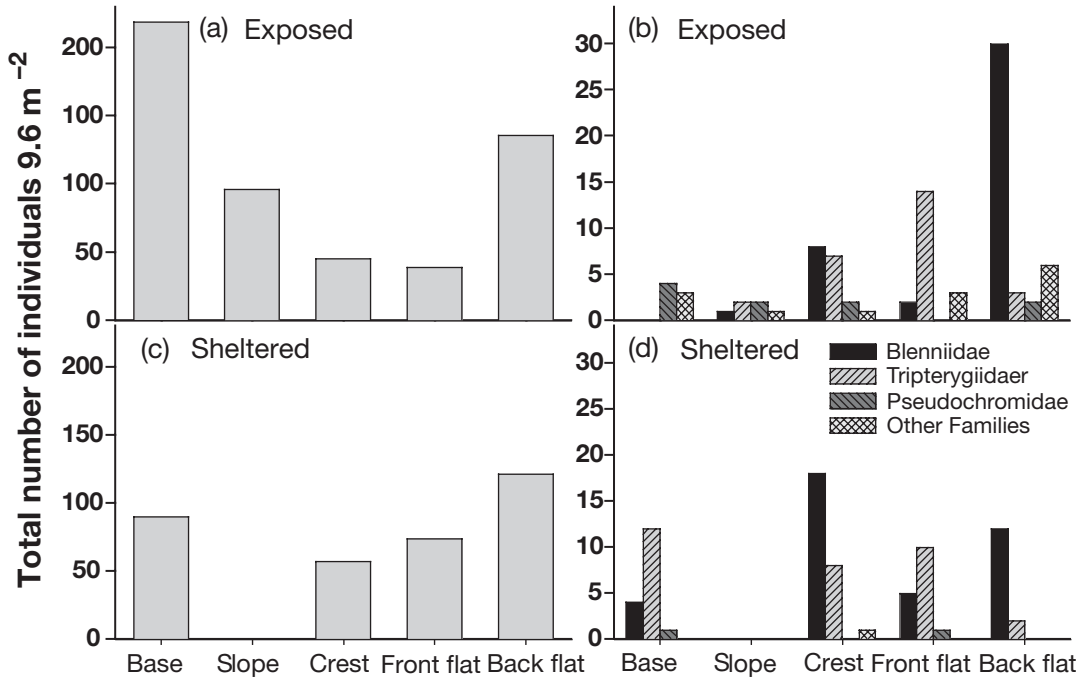

Fig. 3. Familial composition of small cryptic reef fishes across reef zones ( $\mathrm{n}=24$ with a total area of $9.6 \mathrm{~m}^{2}$ per zone). (a,c) Gobiidae, (b,d) non-gobiid families. No samples were taken from the slope at sheltered reef sites. The identity of 'Other Families' is listed in Table 2

Among reef zones, however, species richness varied considerably at the exposed reef, less so at the sheltered reef. With 2 exceptions, where abundances were at their lowest (front flat at exposed reef and crest at sheltered reef), the influence of microhabitat on number of individuals and species is consistent (Figs. 4 \& 5, Table 4) with the sand/rubble microhabitat samples having more individuals and species than open reef microhabitat samples. A total of $68.5 \%$ of all individu-

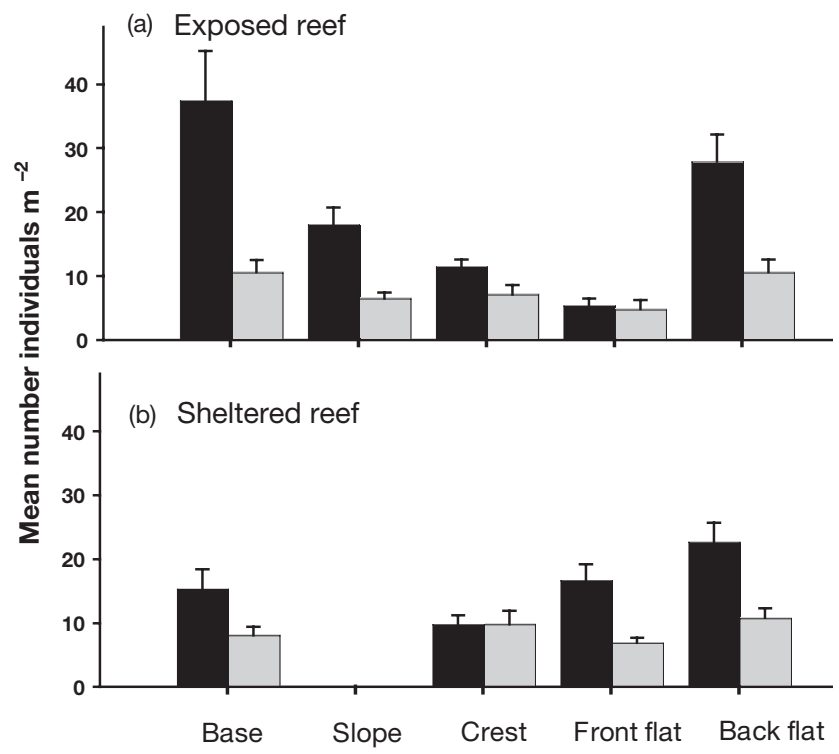

Fig. 4. Mean number of individuals $\mathrm{m}^{-2}(+\mathrm{SE}, \mathrm{n}=12$ samples) among the reef zones and microhabitats sampled at (a) exposed and (b) sheltered reefs. Black bars: sand/rubble; gray bars: open reef microhabitats. No samples were taken from the slope at sheltered reef sites als were censused from sand/rubble habitats and 41 of 44 species. In contrast, open reef samples contained only 27 species in total.

\section{Exposed reef}

Individual abundance and species richness varied significantly at both the zone and microhabitat level (Table 4). The influence of microhabitat on numbers of individuals is apparent for most zones with the exception of the front flat where very low abundances were recorded in both microhabitat types (Fig. 4a). Sand/rubble microhabitats contained $70.1 \%$ of all sampled individuals on exposed reefs with distinct differences between zones, whereas open reef microhabitats were roughly equal in fish abundance across reef zones.

For reef zones, front flat areas were clearly the most depauperate in terms of individuals and species with base and back flat areas the most populated. A Tukey's post-hoc test identified the front flat as being statistically different from all others for both numbers of individuals and species (Table 5); all other reef zones shared varying relationships to one another. In total, 58 individuals and 10 species were found in the

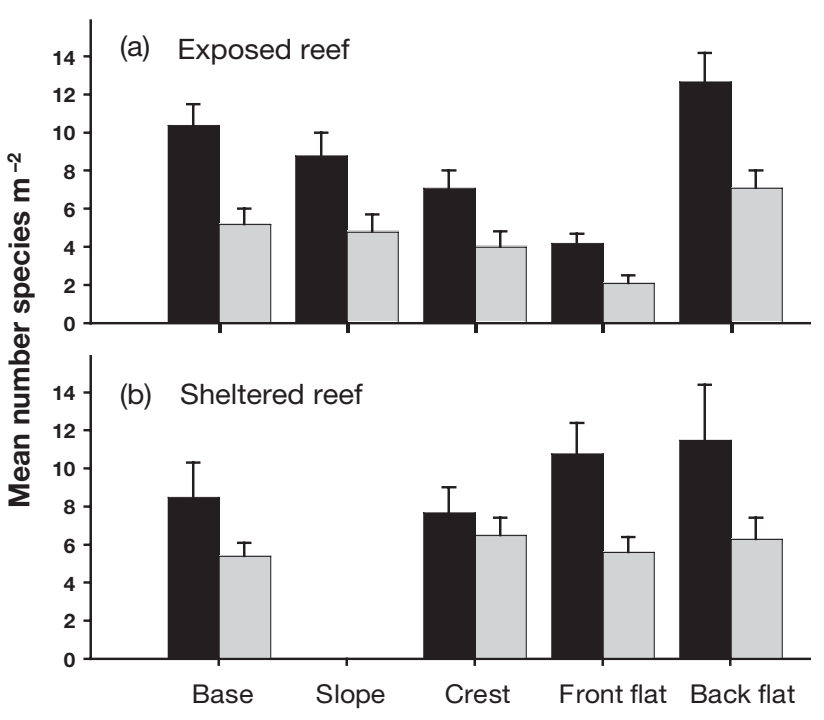

Fig. 5. Mean number of species $\mathrm{m}^{-2}(+\mathrm{SE} ; \mathrm{n}=12$ samples $)$ among the reef zones and microhabitats sampled at (a) exposed and (b) sheltered reefs. Black bars: sand/rubble; gray bars: open reef microhabitats. No samples were taken from the slope at sheltered reef sites 
Table 3. Mean fish density, species richness and mean number of individuals per species $( \pm \mathrm{SE}$ ) for exposed and sheltered reefs for each reef zone. Total number of species found at exposed and sheltered reef sites indicated in bold

\begin{tabular}{|lrrrr|}
\hline Zone & $\begin{array}{c}\text { No. of } \\
\text { ind. }\end{array}$ & $\begin{array}{c}\text { Density } \\
\left(\mathrm{m}^{-2}\right)\end{array}$ & $\begin{array}{c}\text { No. of } \\
\text { species/zone }\end{array}$ & $\begin{array}{c}\text { Mean no. } \\
\text { ind./species }\end{array}$ \\
\hline Exposed & & & & \\
Base & 224 & 23.4 & 13 & $16.1( \pm 9.6)$ \\
Slope & 105 & 10.9 & 14 & $7.5( \pm 2.9)$ \\
Crest & 63 & 6.5 & 18 & $3.5( \pm 0.9)$ \\
Front flat & 58 & 6.0 & 10 & $5.8( \pm 2.1)$ \\
Back flat & 176 & 18.3 & 20 & $8.8( \pm 4.0)$ \\
Total & $\mathbf{6 2 6}$ & & $\mathbf{3 1}$ & \\
Sheltered & & & & \\
Base & 96 & 10.0 & 22 & $4.5( \pm 1.0)$ \\
Crest & 80 & 8.3 & 27 & $3.1( \pm 0.7)$ \\
Front flat & 99 & 10.3 & 17 & $5.4( \pm 1.4)$ \\
Back flat & 141 & 14.7 & 17 & $8.4( \pm 2.8)$ \\
Total & $\mathbf{4 1 6}$ & & $\mathbf{3 6}$ & \\
\hline
\end{tabular}

front flat zone compared to 176 individuals and 20 different species in back flat zone (Table 3).

\section{Sheltered reef}

Fish abundance and species richness patterns showed comparatively little among-reef zone variation in sheltered reefs (Figs. 4b \& 5b). A significant site $\times$ zone $\times$ microhabitat interaction indicates that patterns
Table 5. Results of Tukey's post-hoc tests identifying the reef zones that statistically differ from each other in individual and species numbers at the exposed reef location. Bold numbers denote significance at $\mathrm{p}<0.05$

\begin{tabular}{|lcccc|}
\hline & Base & Slope & Crest & Front flat \\
\hline No. of individuals & & & & \\
Base & & & & \\
Slope & $\mathbf{0 . 0 2 4}$ & & & \\
Crest & $\mathbf{0 . 0 0 2}$ & 0.933 & & \\
Front flat & $\mathbf{0 . 0 0 0}$ & $\mathbf{0 . 0 0 0}$ & $\mathbf{0 . 0 0 1}$ & \\
Back flat & 1.0 & $\mathbf{0 . 0 2 1}$ & $\mathbf{0 . 0 0 2}$ & $\mathbf{0 . 0 0 0}$ \\
No. of species & & & & \\
Base & & & & \\
Slope & 0.783 & & & \\
Crest & 0.270 & 0.909 & & \\
Front flat & $\mathbf{0 . 0 0 0}$ & $\mathbf{0 . 0 0 3}$ & $\mathbf{0 . 0 3 6}$ & $\mathbf{0 . 0 0 0}$ \\
Back flat & 0.490 & 0.053 & $\mathbf{0 . 0 0 4}$ & $\mathbf{0 . 0 0 0}$ \\
\hline
\end{tabular}

of abundance at this sheltered reef may be quite complex in comparison to those found at the exposed reef (Table 4). A more thorough investigation indicated that statistical differences were primarily driven by the microhabitat and, to a lesser extent, reef zone factors. Inconsistent patterns exist among and within the 3 factors aside from a trend towards higher abundances in back flat reef zones and sand/rubble microhabitats. A Tukey's post-hoc test identified differences between the back flat and all other reef zones as the major determinant of statistical differences for abundance at the reef zone level (Table 6).
Table 4. Three-way ANOVA results comparing fish abundance and species richness $\left[\log _{10}(x+1)\right]$ in exposed and sheltered reefs amongst sites, reef zones and microhabitats (M'habitat). Exposed reef location: $\mathrm{n}=120, \mathrm{df}=4$;

Sheltered: $\mathrm{n}=96, \mathrm{df}=3$. Bold numbers denote significance at $\mathrm{p}<0.05$

\begin{tabular}{|lrrrrrr|}
\hline \multirow{2}{*}{ Source } & \multicolumn{3}{c}{ Fish abundance } & \multicolumn{3}{c}{ Species richness } \\
& MS & \multicolumn{1}{c}{$F$} & $\mathrm{p}$ & $\mathrm{MS}$ & $F$ & $\mathrm{p}$ \\
\hline Exposed reef & & & & & & \\
Site & 0.14 & 2.51 & 0.087 & 0.02 & 0.07 & 0.934 \\
Zone & 1.15 & 21.07 & $\mathbf{0 . 0 0 1}$ & 0.40 & 11.93 & $<\mathbf{0 . 0 0 1}$ \\
M'habitat & 2.73 & 50.18 & $\mathbf{< 0 . 0 0 1}$ & 1.12 & 33.12 & $<\mathbf{0 . 0 0 1}$ \\
Site $\times$ Zone & 0.10 & 1.74 & 0.099 & 0.04 & 1.07 & 0.389 \\
Site $\times$ M'habitat & 0.01 & 0.25 & 0.776 & 0.01 & 0.40 & 0.672 \\
Zone $\times$ M'habitat & 0.08 & 1.39 & 0.244 & 0.01 & 0.21 & 0.932 \\
Site $\times$ Zone $\times$ M'habitat & 0.05 & 0.88 & 0.540 & 0.02 & 0.54 & 0.821 \\
Error & 0.05 & & & 0.03 & & \\
Sheltered reef & & & & & & \\
Site & & & & & & \\
Zone & 0.09 & 2.64 & 0.078 & 0.05 & 1.99 & 0.144 \\
M'habitat & 0.16 & 4.96 & $\mathbf{0 . 0 0 3}$ & 0.04 & 1.30 & 0.283 \\
Site $\times$ Zone & 0.85 & 25.87 & $\mathbf{0 . 0 0 1}$ & 0.44 & 16.14 & $<\mathbf{0 . 0 0 1}$ \\
Site $\times$ M'habitat & 0.06 & 1.71 & 0.130 & 0.05 & 1.66 & 0.143 \\
Zone $\times$ M'habitat & 0.11 & 3.22 & $\mathbf{0 . 0 4 6}$ & 0.09 & 3.42 & $\mathbf{0 . 0 3 8}$ \\
Site $\times$ Zone $\times$ M'habitat & 0.10 & 3.05 & $\mathbf{0 . 0 3 4}$ & 0.05 & 1.64 & 0.187 \\
Error & 0.10 & 2.99 & $\mathbf{0 . 0 1 2}$ & 0.06 & 2.20 & 0.053 \\
& 0.03 & & & 0.03 & & \\
\hline
\end{tabular}

\section{Patterns in size}

Total lengths of individuals varied from 7.5 to $92.6 \mathrm{~mm}$ TL overall, with $91 \%$ of all individuals measuring between 7.5 to $29.9 \mathrm{~mm}$ (mean $19.1 \pm 0.3 \mathrm{SE}$ ). Striking differences in the mean weight of individuals were apparent at exposed sites $\left(F_{4,626}=13.27, \mathrm{p}<0.001\right)$ with the heaviest individuals coming from exposed waveswept reef zones (crest, front flat and back flat) (Fig. 6a). Individuals present at the front flat reef zone had mean weights of $0.34 \mathrm{~g}( \pm 0.13 \mathrm{SE})$; at the crest of $0.18 \mathrm{~g}$ $( \pm 0.04 \mathrm{SE}) ;$ and at the back flat of $0.14 \mathrm{~g}$ $( \pm 0.02 \mathrm{SE})$. These values are in stark contrast to the $0.07 \mathrm{~g}( \pm 0.01 \mathrm{SE})$ in base and slope reef zones (Fig. 6a). Tukey's posthoc tests differentiate these 2 groups. While half of all individuals of $<15 \mathrm{~mm}$ at the exposed reef sites were found at the base, the largest individuals (>45 mm) were predominantly found at crest and 
Table 6. Results of Tukey's post-hoc tests identifying the reef zones that statistically differ from each other in individual and species numbers at the sheltered reef location. Bold numbers denote significance at $\mathrm{p}<0.05$

\begin{tabular}{|lccc|}
\hline & Base & Crest & Front flat \\
\hline No. of individuals & & & \\
Base & & & \\
Crest & 0.833 & & \\
Front flat & 0.998 & 0.742 & \\
Back flat & $\mathbf{0 . 0 3 3}$ & $\mathbf{0 . 0 0 3}$ & $\mathbf{0 . 0 4 9}$ \\
No. of species & & & \\
Base & & & \\
Crest & 0.987 & & \\
Front flat & 0.589 & 0.790 & \\
Back flat & 0.313 & 0.504 & 0.965 \\
\hline
\end{tabular}

back flat reef zones. Of these, approximately half were from the family Blenniidae, with larger gobies (Amblygobius phaelena, A. rainfordi, Valenciennea muralis and Istigobius goldmanni) making up the remainder. Reef zones at the sheltered sites were less variable, but overall differences were significant $\left(F_{3,416}=15.11, \mathrm{p}<\right.$ 0.001). This pattern is due to the crest zone where mean individual weights $(0.32 \mathrm{~g} \pm 0.04 \mathrm{SE})$ were nearly twice that of the overall mean across all reef zones (0.18 $g, \pm 0.14 \mathrm{SE}$ ) (Fig. 6b). For these sites, the crest contained the highest abundance of the $>45 \mathrm{~mm}$ size-class, due to the presence of large blenniid species in the crest reef zone. Size-classes were evenly spread across the other 3 sheltered reef zones.

\section{Patterns in species assemblages}

Only 6 species (from the 12 selected for analyses) were abundant (with $>10$ individuals) at both exposed and sheltered reef sites; Eviota melasma, E. sigillata, E. queenslandica, Istigobius goldmanni, Enneapterygius tutuilae and Salarias patzneri. Callogobius sp. A and Ctenogobiops feroculus were found exclusively at sheltered reef sites; Eviota sp. J and E. pellicuda were only found on exposed reef sites. MANOVAs based on the 12 most abundant species at exposed and at sheltered reef sites revealed significant differences in species assemblages among reef zones for both exposed and sheltered reefs (Pillai's trace $\mathrm{p}<0.001$ ). Post-hoc Bonferroni-corrected multiple

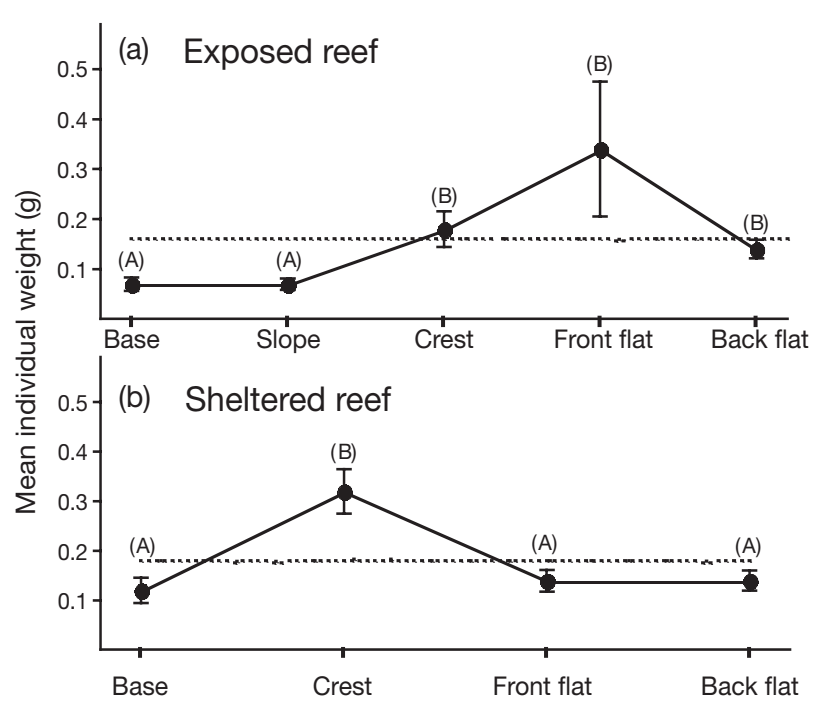

Fig. 6. Mean weight $(\mathrm{g})( \pm \mathrm{SE}, \mathrm{n}=24$ samples $)$ of all individuals censused at (a) exposed and (b) sheltered reef zones. Dotted line represents the average weight $(\mathrm{g})$ across all reef zones for exposed and sheltered reef sites. Letters denote statistically indistinguishable groupings (same letter)

comparisons indicated that species abundances varied among reef zones in 10 of 12 species at exposed reef sites, and 6 of 12 at sheltered reef sites (Table 7). Canonical discriminant analysis shows the nature of

Table 7. Results of the Bonferroni-corrected multiple comparisons tests identifying the species that differed significantly in abundance among reef zones at exposed and sheltered reef sites. Analyses was based on the 12 most abundant ( $>10$ individuals across all zones) species censused at (1) exposed and, (2) sheltered reef sites. Letters indicate statistical non-significance (same letter) or significant differences (different letter) of each species among relevant reef zones. No samples were taken from the slope at sheltered reef sites

\begin{tabular}{|lccccccc|}
\hline & Base & Slope & Crest & $\begin{array}{c}\text { Front } \\
\text { flat }\end{array}$ & $\begin{array}{c}\text { Back } \\
\text { flat }\end{array}$ & $p$ \\
\hline Exposed reef & & & & & & \\
Eviota melasma & & & & & & \\
Eviota sp. J & $\mathrm{A}$ & $\mathrm{A}$ & $\mathrm{B}$ & $\mathrm{B}$ & $\mathrm{B}$ & $<0.001$ \\
Eviota sigillata & $\mathrm{A}$ & $\mathrm{B}$ & $\mathrm{B}$ & $\mathrm{B}$ & $\mathrm{B}$ & $<0.001$ \\
Eviota sp. O & $\mathrm{A}$ & $\mathrm{A}, \mathrm{B}$ & $\mathrm{B}$ & $\mathrm{B}$ & $\mathrm{B}$ & $<0.001$ \\
Eviota queenslandica & $\mathrm{A}$ & $\mathrm{A}$ & $\mathrm{B}$ & $\mathrm{A}$ & $\mathrm{A}$ & $<0.01$ \\
Eviota pellicuda & $\mathrm{A}$ & $\mathrm{A}$ & $\mathrm{A}$ & $\mathrm{A}$ & $\mathrm{B}$ & $<0.001$ \\
Istigobius goldmanni & $\mathrm{A}, \mathrm{C}$ & $\mathrm{A}$ & $\mathrm{B}, \mathrm{C}$ & $\mathrm{B}, \mathrm{C}$ & $\mathrm{B}, \mathrm{C}$ & $<0.05$ \\
Enneapterygius tutuilae & $\mathrm{A}$ & $\mathrm{A}$ & $\mathrm{A}$ & $\mathrm{A}$ & $\mathrm{B}$ & $<0.001$ \\
Fusigobius signipinnis & $\mathrm{B}$ & $\mathrm{B}$ & $\mathrm{A}, \mathrm{B}$ & $\mathrm{A}$ & $\mathrm{A}, \mathrm{B}$ & $<0.01$ \\
Salarias patzneri & $\mathrm{A}, \mathrm{B}$ & $\mathrm{A}$ & $\mathrm{A}, \mathrm{B}$ & $\mathrm{B}$ & $\mathrm{B}$ & $<0.05$ \\
Sheltered reef & $\mathrm{A}$ & $\mathrm{A}$ & $\mathrm{A}$ & $\mathrm{A}$ & $\mathrm{B}$ & $<0.001$ \\
Eviota melasma & & & & & & \\
Eviota queenslandica & $\mathrm{A}$ & - & $\mathrm{B}$ & $\mathrm{B}$ & $\mathrm{B}$ & $<0.001$ \\
Asterropteryx semipunctatus & $\mathrm{A}$ & - & $\mathrm{B}$ & $\mathrm{B}$ & $\mathrm{A}$ & $<0.001$ \\
Istigobius goldmanni & $\mathrm{B}$ & - & $\mathrm{A}, \mathrm{C}$ & $\mathrm{A}, \mathrm{C}$ & $\mathrm{B}, \mathrm{C}$ & $<0.05$ \\
Salarias patzneri & $\mathrm{B}$ & - & $\mathrm{A}$ & $\mathrm{A}, \mathrm{B}$ & $\mathrm{A}$ & $<0.001$ \\
Ctenogobiops feroculus & $\mathrm{B}$ & - & $\mathrm{A}, \mathrm{B}$ & $\mathrm{A}, \mathrm{B}$ & $\mathrm{B}$ & $<0.01$ \\
& & & $\mathrm{~A}$ & $<0.05$ \\
\hline
\end{tabular}



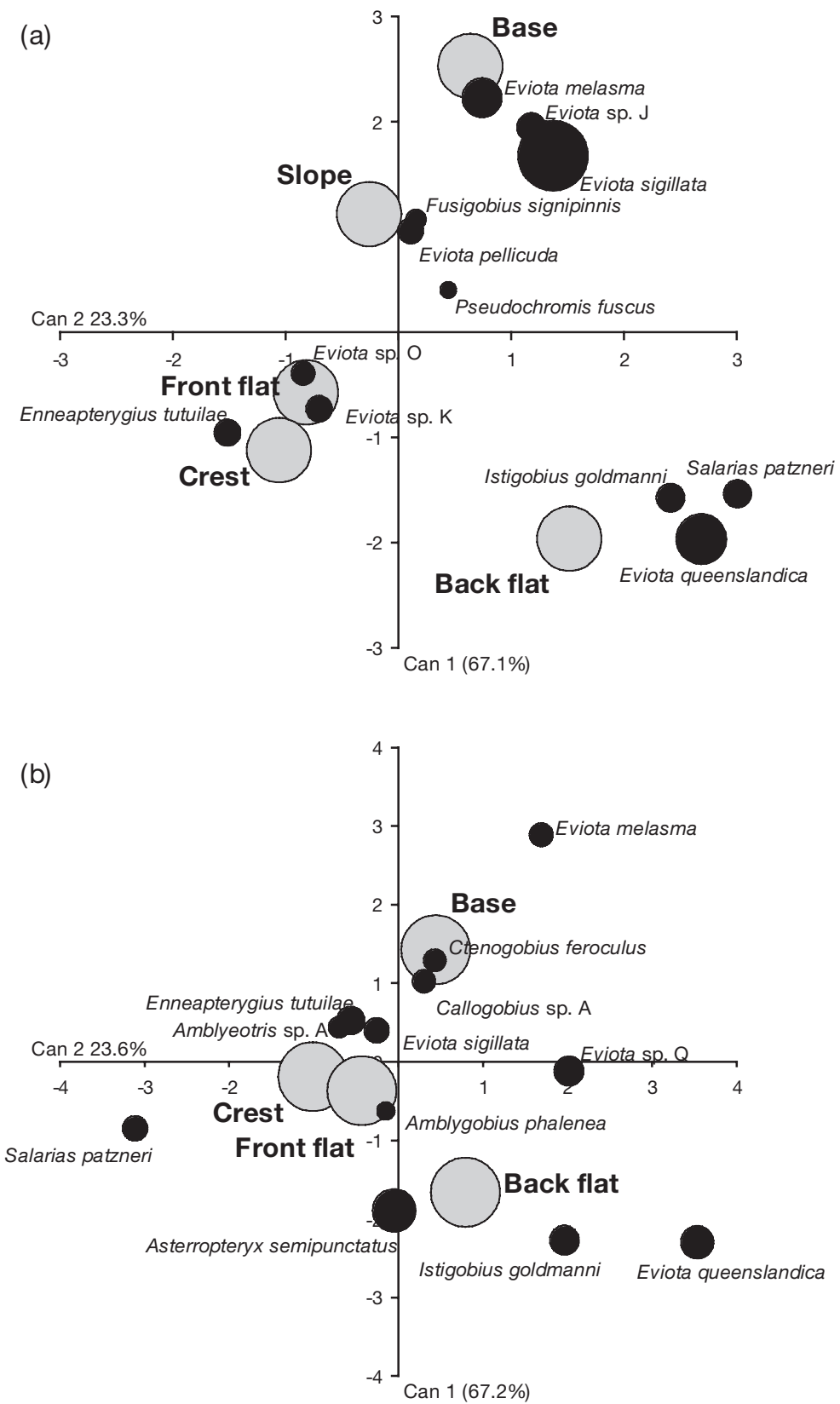

Fig. 7. Canonical discriminant analyses (CDA) displaying the relationship between the small cryptic reef fish community (12 most abundant species) and reef zones at (a) exposed and (b) sheltered reef sites. Confidence clouds (95\%) surround reef zone centroids (gray circles). Species bubble sizes reflect total abundance of each species (square root transformation from raw counts)

\section{DISCUSSION}

Our analysis of the small cryptic reef fish community at Lizard Island revealed 3 clear trends. Firstly, sand/rubble microhabitats consistently supported more individuals and species than open reef microhabitats, regardless of the location or spatial scale examined. Secondly, although community patterns of abundance, diversity and size/weight-class distribution varied widely among zones at exposed sites, comparatively little variation was exhibited at sheltered sites. Thirdly, species composition varied considerably along wave energy gradients between exposed and sheltered reef sites and among reef zones.

With the exception of depauperate front flat (exposed sites) and crest (sheltered sites) reef zones, the effect of microhabitat type on the distribution patterns of the small reef fish community at Lizard Island was clear; more than two thirds of individuals and 41 out of a possible 44 species were sampled on sand/ rubble microhabitats as opposed to 27 species in open reef samples. Intuitively, the relationship between small substratum-bound fishes and their structural environment is likely to be an intimate one, and it has already been well established that microhabitat type plays a pivital role in the survival, abundance and distribution of these assemblages at small spatial scales (Syms 1995, Prochazka 1998, Munday 2000, Wilson 2001, Willis \& Anderson 2003, La Mesa et al. 2004). At this scale, levels of abundance and species richness increase dramatically in more heterogenous environments that offer quality shelter to residents (Caley \& St John 1996, Willis \& Anderson 2003, Depczynski \& Bellwood 2004). Given that microhabit type plays such a key role in structuring small reef fish communities, the question remains,

these differences where centroid means and 95\% confidence clouds are plotted for each zone at exposed (Fig. 7a) and sheltered (Fig. 7b) reefs. Except for the front flat and crest, confidence clouds indicate that all other zones have characteristic species assemblages in both exposed and sheltered reef sites. Small, highly abundant species tended to be associated with base and slope zones at exposed reef sites. to what extent do larger scale habitat zones influence community distribution structure?

At exposed wave-swept reef zones, we found a dramatic decrease in diversity and abundance, with a corresponding increase in overall fish size. This was most marked in the shallow wave-swept front flat, and to a lesser extent reef crest zones, regardless of microhabitat type. This suggests that microhabitat type 
plays a minor or secondary role in structuring small reef fish communities under circumstances where incident wave energy is particularly strong. There are 2 , non-exclusive factors that may account for these patterns. Firstly, wave-induced water motion may directly affect the distribution of many reef fish species through its interaction with swimming performance. Secondly, wave energy influences their distribution through change to benthic communities and habitat structure.

The swimming abilities of small cryptic coral reef fishes have yet to be quantified. In larger, more mobile species a direct relationship between wave-induced water motion and swimming mode has been identified for a number of reef fish families (Fulton \& Bellwood 2005). It appears that wave-swept habitats may have high energetic costs of occupation which may be a barrier for some species based on their swimming mode and efficiency. Fishes with sustained swimming abilities, usually employing energy-efficient lift-based pectoral locomotion, tend to inhabit wave exposed reef environments while slower thrust-based swimmers occupy more sheltered environments (Bellwood \& Wainwright 2001, Fulton \& Bellwood 2005). Several lines of evidence suggest that small cryptic fishes are comparatively poor swimmers. Unlike larger, more mobile species, most small cryptic species are substratumbound (many lacking a swim bladder), spending little or none of their time swimming high in the water column where water movement may be greatest (Shashar et al. 1996, Goncalves \& Almada 1998). Furthermore, they lead spatially restrictive lives encompassing home ranges of 0.25 to $2 \mathrm{~m}^{2}$ and many tend to exhibit quite sedentary behaviour (Luckhurst \& Luckhurst 1978, Goncalves \& Almada 1998, Depczynski \& Bellwood 2004). Small cryptic reef fish swimming generally consists of short bursts (1 to $5 \mathrm{~s}$ ) using body and caudal fin propulsion which may be one of the most energetically expensive modes (Wu 1977, Vogel 1994). Furthermore, their predominantly rounded fins suggest they are suited to powerful short-bursts of speed rather than sustained high speed swimming (Sambilay 1990, Vogel 1994). Overall, it would appear that the swimming ability of small cryptic species are generally unsuitable for high energy wave-swept locations.

Despite these limitations, our results indicate that a few species are able to inhabit even severely wave affected reef areas (i.e. families Blenniidae and Tripterygiidae). Size distributions among exposed reef zones provided some interesting insights into the potential role of wave energy and water motion in structuring size-related spatial patterns in these small reef fish communities. Our data show that, at exposed reef sites, wave-swept reef zones (i.e. front flat) were inhabited by low numbers of larger, heavier (25 to $93 \mathrm{~mm}$ TL) individuals including species with high sur- face area:volume ratios such as blennies, and calm reef zones (i.e. base) by high numbers of very small, lighter ( $<15 \mathrm{~mm}$ TL) ones. These results correlate well with previously described patterns in the Caribbean (Greenfield \& Johnson 1981, Greenfield 2003) and at Lizard Island (Wilson 2001) where some species of Blenniidae appear to show preferences for shallow, high energy habitats. Differences in size-class distributions across wave exposure gradients have previously been documented in temperate labrids with smilar results to those presented here (Fulton \& Bellwood 2004). Shallow, wave-exposed reef zones were not only poorly inhabited by fewer and larger individuals, but smaller individual size-classes were almost entirely absent. In an earlier paper, Fulton \& Bellwood (2002) also demonstrated ontogenetic changes in water column use for coral reef wrasses, with smaller individuals remaining close to the substratum, the authors hypothesising that smaller, less competent swimmers were flow-refuging in near-bottom boundary layers or microhabitat scale eddies. Regardless of the underlying mechanisms, the ability to maintain station in these hostile environments is probably very difficult for small individuals and our data suggests that waveswept environments may provide a serious challenge to their occupation by smaller-sized individuals.

The role of wave energy in shaping marine environments and marine communities has been well documented for many marine ecosystems (Menge 1976, McQuaid \& Branch 1985, Denny 1988, Friedlander \& Parrish 1998, Denny \& Wethey 2001, Bellwood et al. 2002, Friedlander et al. 2003). Because coral reef ecosystems are biogenic in makeup, presence, absence, type and morphology of reef building organisms are greatly influenced by wave induced water motion (Dineson 1983, Done 1983, Ninio \& Meekan 2002), shaping the habitat and living areas of resident biota. Distinct and abrupt changes in reef habitat diversity, complexity and abundance coinciding with changes from one reef zone to another follow depth and corresponding wave energy gradients. The microhabitat at the front flat is essentially level homogenous algal encrusted rock pavement subjected to the highest levels of water motion in our study. Accordingly, open reef microhabitats on exposed wave-swept reef flats theoretically represent the most physically extreme location for small benthic fishes and we found these areas to be poorly inhabited. At our sheltered reef sites where more gradual inter-zone changes take place, we see little overall variation in these community parameters. These differences in habitat topography may be a significant influence on small cryptic reef fish communities.

Trophic resource patterns may also be a significant factor in shaping cryptic reef fish distributions. Most 
small cryptic reef fishes on the Great Barrier Reef are detritivorous (Depczynski \& Bellwood 2003). Despite the higher nutritional value of detritus from exposed reef crests (Purcell \& Bellwood 2001), loose detrital aggregates settle and concentrate in habitats and reef areas of low water movement such as lagoonal back reefs (Koop \& Larkum 1987, Hansen et al. 1992) where they are easily accessed by benthic feeders. For detritivorous species which are physically able to cope with higher water velocities, such as Ctenochaetus species (Acanthuridae), reef crests and flats are highly productive and nutritionally rich reef zones and represent preferred locations (Choat \& Bellwood 1985). For small cryptic reef fishes, however, these zones are mainly restricted to larger species.

In summary, we find that small cryptic reef fish taxa show a strong level of among-zone variation on coral reefs, but only in locations with high water movement. Microhabitat plays a consistent but secondary role. Regardless of the mechanism, whether direct through water movement impacts on swimming or indirect through habitat or food availability, water movement appears to be a significant factor shaping small cryptic coral reef fish communities.

Acknowledgements. We thank A. Barnett and the staff at Lizard Island Research Station for field support; A. Hoey and T. Hancock for statistical advice; H. Larson and P. Munday for helpful discussions and taxonomic identifications; C. Fulton, M. Gagliano and A. Hoey for constructive comments on the manuscript. This research was funded by the Australian Research Council (D.R.B.), a Reef CRC Augmentative grant (M.D.), and James Cook University. JCU Experimentation Ethics Committee approval \#A787_02. Centre for Coral Reef Biodiversity publication \#148.

\section{LITERATURE CITED}

Ackerman JL, Bellwood DR (2000) Reef fish assemblages: a re-evaluation using enclosed rotenone stations. Mar Ecol Prog Ser 206:227-237

Ackerman JL, Bellwood DR (2002) Comparative efficiency of clove oil vs rotenone for sampling tropical reef fish assemblages. J Fish Biol 60:893-901

Begon M, Harper JL, Townsend CR (1996) Ecology; individuals, populations and communities, 3rd edn. Blackwell Science, Carlton

Bellwood DR, Wainwright PC (2001) Locomotion in labrid fishes: implications for habitat use and cross-shelf biogeography on the Great Barrier Reef. Coral Reefs 20:139-150

Bellwood DR, Wainwright PC, Fulton CJ, Hoey A (2002) Assembly rules and functional groups at global biogeographical scales. Funct Ecol 16:557-562

Brock RE (1982) A critique of the visual census method for assessing coral reef fish populations. Bull Mar Sci 32: 269-276

Caley JM, St John J (1996) Refuge availability structures assemblages of tropical reef fishes. J Anim Ecol 65:414-428

Choat JH, Bellwood DR (1985) Interactions amongst herbivorous fishes on a coral reef: influence of spatial variation. Mar Biol 89:221-234
Denny MW (1988) Biology and the mechanics of the waveswept environment. Princeton University Press, Princeton, NJ

Denny M, Wethey D (2001) Physical processes that generate patterns in marine communities. In: Bertness MD, Gaines SD, Hay ME (eds) Marine community ecology. Sinauer Associates, Sunderland, MA, p 3-37

Depczynski M, Bellwood DR (2003) The role of cryptobenthic reef fishes in coral reef trophodynamics. Mar Ecol Prog Ser 256:183-191

Depczynski M, Bellwood DR (2004) Microhabitat utilization patterns in cryptobenthic coral reef fish communities. Mar Biol 145:455-463

Depczynski M, Bellwood DR (2005) Shortest recorded vertebrate lifespan found in a coral reef fish. Curr Biol 15(8): R288-R289

Dineson ZD (1983) Patterns in the distribution of soft corals across the central Great Barrier Reef. Coral Reefs 1: $229-236$

Done TJ (1983) Coral zonation: its nature and significance. In: Barnes DJ (ed) Perspectives on coral reefs. Australian Institute of Mariune Science, Townsville, p 107-147

Edgar GJ, Barrett NS, Morton AJ (2004) Biases associated with the use of underwater visual census techniques to quantify the density and size-structure of fish populations. J Exp Mar Biol Ecol 308:269-290

Friedlander AM, Parrish JD (1998) Habitat characteristics affecting fish assemblages on a Hawaiian reef. J Exp Mar Biol Ecol 224:1-30

Friedlander AM, Brown EK, Jokiel PL, Smith WR, Rodgers KS (2003) Effects of habitat, wave exposure, and marine protected area status on coral reef fish assemblages in the Hawaiian archipelago. Coral Reefs 22:291-305

Fulton CJ, Bellwood DR (2002) Ontogenetic habitat use in labrid fishes: an ecomorphological perspective. Mar Ecol Prog Ser 236:255-262

Fulton CJ, Bellwood DR (2004) Wave exposure, swimming performance, and the structure of tropical and temperate reef fish assemblages. Mar Biol 144:429-437

Fulton CJ, Bellwood DR (2005) Wave-induced water motion and the functional implications for coral reef fish assemblages. Limnol Oceanogr 50:255-264

Fulton CJ, Bellwood DR, Wainwright PC (2001) The relationship between swimming ability and habitat use in wrasses (Labridae). Mar Biol 139:25-33

Goncalves EJ, Almada VC (1998) A comparative study of territoriality in intertidal and subtidal blennioids (Teleostei, Blennioidei). Environ Biol Fish 51:257-264

Goncalves EJ, Barbosa M, Cabral HN, Henriques M (2002) Ontogenetic shifts in patterns of microhabitat utilization in the small-headed clingfish, Apletodon dentatus (Gobiesocidae). Environ Biol Fish 63:333-339

Greenfield DW (2003) A survey of the small reef fishes of Kane'ohe Bay O'ahu, Hawaiian Islands. Pac Sci 57:45-76

Greenfield DW, Johnson RK (1981) The blennioid fishes of Belize and Honduras, central America, with comments on their systematics, ecology, and distribution (Blenniidae, Chaenopsidae, Labrisomidae, Tripterygiidae). Fieldiana Zool New Ser 8:1-106

Greenfield DW, Johnson RK (1990) Community structure of western Caribbean blennioid fishes. Copeia 1990:433-448

Greenfield DW, Johnson RK (1999) Assemblage structure and habitat associations of western Caribbean gobies (Teleostei: Gobiidae). Copeia 1999:251-266

Gust N, Choat JH, McCormick MI (2001) Spatial variability in reef fish distribution, abundance, size and biomass: a multi-scale analysis. Mar Ecol Prog Ser 214:237-251 
Hansen JA, Klumpp DW, Alongi DM, Dayton PK, Riddle MJ (1992) Detrital pathways in a coral reef lagoon. Mar Biol 113:363-372

Hobbs JPA, Munday PL (2004) Intraspecific competition controls spatial distribution and social organisation of the coral-dwelling goby Gobiobon histrio. Mar Ecol Prog Ser 278:253-259

Koop K, Larkum AWD (1987) Deposition of organic material in a coral reef lagoon, One Tree Island, Great Barrier Reef. Estuar Coast Shelf Sci 25:1-9

Lachner EA, Karnella SJ (1980) Fishes of the Indo-Pacific genus Eviota with descriptions of eight new species (Teleostei: Gobiidae). Smithson Contrib Zool 315:1-127

La Mesa G, Micalizzi M, Giaccone G, Vacchi M (2004) Cryptobenthic fishes of the Ciclopi Islands marine reserve (central Mediterranean Sea): assemblage composition, structure and relations with habitat features. Mar Biol 145: 233-242

Luckhurst BE, Luckhurst K (1978) Diurnal space utilisation in coral reef fish communities. Mar Biol 49:325-332

May RM (1978) The dynamics and diversity of insect faunas. In: Mound LA, Walcoff N (eds) Diversity of insect faunas. Symposia of the Royal Entomological Society of London, Blackwell Scientific Publications, Oxford, p188-294

McQuaid CD, Branch GM (1985) Trophic structure of rocky intertidal communities: Response to wave action and implications for energy flow. Mar Ecol Prog Ser 22: 153-161

Menge BA (1976) Organization of the New England rocky intertidal community: role of predation, competition, and environmental heterogeneity. Ecol Monogr 46:355-393

Munday PL (2000) Interactions between habitat use and patterns of abundance in coral-dwelling fishes of the genus Gobiodon. Environ Biol Fish 58:355-369

Munday PL, Pierce SJ, Jones GP, Larson HK (2002) Habitat use, social organization and reproductive biology of the seawhip goby, Bryaninops yongei. Mar Freshw Res 53: 757-768

Ninio R, Meekan MG (2002) Spatial patterns in benthic communities and the dynamics of a mosaic ecosystem on the Great Barrier Reef, Australia. Coral Reefs 21:95-103

Prochazka K (1998) Spatial and trophic partitioning in cryptic fish communities of shallow subtidal reefs in False Bay, South Africa. Environ Biol Fish 51:201-220

Editorial responsibility: Charles Birkeland (Contributing Editor), Honolulu, Hawaii, USA
Purcell SW, Bellwood DR (2001) Spatial patterns of epilithic algal and detrital resources on a windward coral reef. Coral Reefs 20:117-125

Reavis RH (1997) The natural history of a monogamous coralreef fish, Valenciennea strigata (Gobiidae):1. Abundance, growth, survival and predation. Environ Biol Fish 49(2): 239-246

Russ GR (1984) Distribution and abundance of herbivorous grazing fishes in the central Great Barrier Reef. II: Patterns of zonation of mid-shelf and outer-shelf reefs. Mar Ecol Prog Ser 20:35-44

Sambilay VCJ (1990) Interrelationships between swimming speed, caudal fin aspect ratio and body length of fishes. Fishbyte 8:16-20

Shashar N, Kinane S, Jokiel PL, Patterson MR (1996) Hydromechanical boundary layers over a coral reef. J Exp Mar Biol Ecol 199:17-28

Syms C (1995) Multi-scale analysis of habitat association in a guild of blennioid fishes. Mar Ecol Prog Ser 125:31-43

Townsend KA, Tibbetts IR (2000) Biomass and distribution of herbivorous blennies in the southern Great Barrier Reef. J Fish Biol 56:774-791

Vogel S (1994) Life in moving fluids: the physical biology of flow, 2nd edn. Princeton University Press, Princeton, NJ

Webster MS, Hixon MA (2000) Mechanisms and individual consequences of intraspecific competition in a coral-reef fish. Mar Ecol Prog Ser 196:187-194

Williams DMcB (1982) Patterns in the distribution of fish communities across the central Great Barrier Reef. Coral Reefs $1: 35-43$

Williams DMcB (1991) Patterns and processes in the distribution of coral reef fishes. In: Sale PF (ed) The ecology of fishes on coral reefs. Academic Press, San Diego, CA, p $437-474$

Willis TJ (2001) Visual census methods underestimate density and diversity of cryptic reef fishes. J Fish Biol 59:1408-1411

Willis TJ, Anderson MJ (2003) Structure of cryptic reef fish assemblages: relationships with habitat characteristics and predator density. Mar Ecol Prog Ser 257:209-221

Wilson S (2001) Multiscale habitat associations of detrivorous blennies (Blenniidae: Salariini). Coral Reefs 20:245-251

Wu TY (1977) Introduction to the scaling of aquatic locomotion. In: Pedley TJ (ed) Scale effects in animal locomotion. Academic Press, London, p 203-232

Submitted: November 29, 2004; Accepted: May 5, 2005

Proofs received from author(s): November 1, 2005 